\title{
A study of laryngeal microsurgery for vocal cord lesions
}

\author{
Shaheen Zafar ${ }^{1}$, Rohit Kumar ${ }^{2}$ \\ ${ }^{1}$ Senior Resident, ${ }^{2}$ PostGraduate Resident, Department of ENT, Patna Medical College and Hospital, Patna, Bihar, India
}

Background: A voice disorder occurs when a person's voice quality, pitch, or volume is out of synch with their age, gender, culture, or location. Moreover, when a person shows concern about an unusual voice that does not satisfy daily necessities, despite the fact that others do not notice, it is considered to have voice problem. Aims and Objectives: Collection and analysis of data base to determine the impact of microlaryngeal surgery (MLS) on voice outcome. Materials and Methods: The study was carried out in the Department of Otorhinolaryngology, in a tertiary care hospital in Patna Medical College andHospital, from October 2020 to June 2021. Study was performed on 30 consecutive cases of benign lesions of vocal cord diagnosed clinically not responding to medical management. Results: Voice handicap index was evaluated preoperatively and postoperatively. The results were compared. Preoperatively, $40 \%$ (12) of patients showed moderate handicap scoring and $60.0 \%$ (18) patients showed severe handicap index. Postoperatively, there was improvement. At $2^{\text {nd }}$, follow-up $33.3 \%(10)$ patients showed moderate handicap scoring, $50.0 \%$ (15) patients showed mild handicap scoring and $16.7 \%$ (5) patients showed severe handicap scoring. At $6^{\text {th }}$ week follow-up $21,(70.0 \%)$ patients showed mild handicap scoring and $30.0 \%$ (9) showed moderate handicap scoring. Finally, at $12^{\text {th }}$ week follow-up, we observed $93.3 \%(28)$ patients had mild handicap scoring while only $6.7 \%(2)$ patients had moderate handicap scoring with significant difference $(P \leq 0.0001)$. Conclusion: MLS is an effective procedure to provide improvement in speech among patients with benign vocal cord lesion.

Key words: Dysphonia; Laryngoscopy; Voice disorders

\section{INTRODUCTION}

The most common cause of dysphonia is benign vocal cord lesions, which can often be cured surgically. Vocal polyps, cysts, nodules, and Reinke'sedema are examples of lesions. They not only affect the patient's ability to correctly pronounce words and speak clearly, but they also cause a number of psychological and social problems, decreasing the patient's quality of life.

Certain laryngeal mucosal problems have been linked to voice abuse, commonly known as phonotrauma. Repetitive impact forces and shear stress produce microvascular damage and trauma to the epithelial basement zone. ${ }^{1}$
Medical therapy, speech language pathology voice therapy, and otolaryngology surgical therapy are all alternatives for treating benign vocal cord abnormalities.

When medical or voice treatment fails to enhance a person's voice, microlaryngeal surgery (MLS) may be required. ${ }^{2}$ The majority of benign vocal cord lesions are treated with MLS to improve voice quality or diagnose the condition. The purpose of MLS is to improve the vibratory qualities of the multilayer microvascular structure of the vocal cord. Stroboscopy is a technique for assessing high-frequency vocal cord vibrations that are difficult to detect with the naked eye. Phonation is created by a cyclic interaction between exhaled air and the fast vocal cord vibrations of the glottic cycle.

\footnotetext{
Address for Correspondence:

Dr.Shaheen Zafar, Senior Resident, Department of ENT, Patna Medical College and Hospital, Patna - 800004 , Bihar, India.

Mobile: +91-9955901433. E-mail: zafaronline1@yahoo.co.in
} 
Proper assessment of these vocal cord oscillations is necessary to describe vocal function and evaluate voice disorders.

Aims and objectives

Collection and analysis of data base to determine the impact of MLS on voice outcome.

\section{MATERIALS AND METHODS}

The study was carried out in the Department of Otorhinolaryngology, in a tertiary care hospital in Patna Medical College andHospital, from October 2020 to June- 2021. Study was performed on 30 consecutive cases of benign lesions of vocal cord diagnosed clinically not responding to medical management. The study was preapproved by Institutional Ethics Committee for the final permission.

\section{Inclusión criteria}

- Cases of benign vocal cord lesions that have been diagnosed clinically but have not responded to medical treatment.

\section{Exclusión criteria}

- Cancer of the larynx.

- Medical treatment alleviated hoarseness symptoms.

- Laryngocele and epiglottic cyst patients.

\section{Methods}

The patients' written informed consents were obtained. Preoperatively, each patient had flexible laryngoscopy, videolaryngoscopy, acoustic analysis, and VHI administration. All of the patients had traditional microlaryngoscopic removal of the benign vocal cord lesion with biopsy under general anesthesia. Videolaryngostroboscopy, acoustic analysis, and VHI were used to examine these patients after 2 weeks, 6 weeks, and 12 weeks after surgery, and the results were recorded.

\section{Videostroboscopy}

Videostroboscopy was performed with the stroboscope system, which includes a $90^{\circ}$ rigid telescope illuminated by a high-performance xenon light for stroboscopy, a microphone mounted on the telescope to record the voice signal from which the fundamental frequency is extracted and used to control the rate of stroboscopic light firing, an endoscope video camera, and a flat screen monitor.

\section{Statistical analysis}

Data werechecked for accuracy and completeness then coded and entered into version 19.0 for analysis. Difference in proportion would be analyzed using Chi-square test, and the difference in means was analyzed using unpaired " $t$ " test. A P $<0.05$ was considered as significant.

\section{RESULTS}

A total of 30 patients were included in the present study. The age ranged between 18 to 55 years with a mean age of 37.41 years. Sex distribution shows a male predominance with $73.3 \%$ male patients and male to female ratio of 2.75:1. 22 patients were presented with unilateral lesion (13, 43.3\%-right sided, 09, 30.0\%-left sided) and 08 (26.7\%) patients with bilateral lesion. $73.3 \%$ of patients presents with hoarseness and $26.7 \%$ with dysphonia. In our study, $60.0 \%$ patients were smokers and $40.0 \%$ were nonsmokers. The benign lesions observed in the study were vocal cord nodules (56.7\%), followed by polyps $(26.7 \%)$, cysts $(10.0 \%)$, andhemangioma on the vocal cord $(6.6 \%)$ (Table1).

Preoperatively the mean fundamental frequency for male patients was found to be 150.23 which showed a statistically significant improvement $(\mathrm{P}=0.002)$ of 176.45 at $12^{\text {th }}$ week of follow-up. However, there was no significant difference in fundamental frequency for females. The shimmer which showed a mean value of 8.95 showed statistically significant $(\mathrm{P}<0.0001)$ improvement to 5.83 . The jitter also showed statistically significant improvement postoperatively, where the pre-operative value was 2.45 and post-operative value was 0.87 . The harmonic to noise ratio value was preoperatively was 11.88 which showed a statistical improvement $(\mathrm{P}<0.0001)$ to 16.10 (Table2).

Voice handicap index was evaluated preoperatively and postoperatively. The results were compared. Preoperatively, $40 \%$ (12) of patients showed moderate handicap scoring and $60.0 \%$ (18) patients showed severe handicap index. Postoperatively, there was improvement. At $2^{\text {nd }}$, follow-up $33.3 \%$ (10) patients showed moderate handicap scoring, $50.0 \%$ (15) patients showed mild handicap scoring and

\begin{tabular}{llll}
\hline Table 1: Demographic variables $(\mathbf{n = 3 0})$ & \\
\hline & Mean and SD & $\begin{array}{c}\text { Number } \\
\text { of cases }\end{array}$ & Percentage \\
\hline Age in Years (18- & $37.41 \pm 11.28$ & & \\
$55)$ & & & \\
Sex & Male & 22 & 73.3 \\
& Female & 08 & 26.7 \\
Side of & Right & 13 & 43.3 \\
involvement & Left & 09 & 30.0 \\
& Bilateral & 08 & 26.7 \\
Clinical & Hoarseness & 22 & 73.3 \\
presentation & Dysphonia & 08 & 26.7 \\
Smoking status & Smokers & 18 & 60.0 \\
& Non-smokers & 12 & 40.0 \\
Types of & Vocal cord & 17 & 56.7 \\
pathology & nodule & & \\
& Vocal cord polyp & 08 & 26.7 \\
& Vocal cord cyst & 03 & 10.0 \\
& Vocal cord & 02 & 6.6 \\
& hemangioma & & \\
\hline
\end{tabular}

Asian Journal of Medical Sciences | Mar 2022 | Vol 13 | Issue 3 
16.7\% (5) patients showed severe handicap scoring. At $6^{\text {th }}$ week follow-up, $21(70.0 \%)$ patients showed mild handicap scoring and $30.0 \%$ (9) showed moderate handicap scoring. Finally, at $12^{\text {th }}$ week follow-up, we observed $93.3 \%$ (28) patients had mild handicap scoring while only $6.7 \%$ (2) patients had moderate handicap scoring with significant difference $(\mathrm{P}<0.0001)$ (Table3).

\section{DISCUSSION}

Medical therapy, voice therapy by speech language pathologists, or surgical therapy by otolaryngologists are all options for treating benign vocal cord abnormalities. ${ }^{2}$ If medical or vocal therapy has failed to enhance a patient's voice, MLS may be required. MLS is used to treat the majority of benign vocal cord lesions, either to restore voice quality or to diagnose the condition. The goal of MLS is to increase the vibratory qualities of the vocal cord's multilayer microvascular structure. ${ }^{3}$

Oertel used stroboscopic examination of the larynx for the first time in 1878. He employed a laryngeal mirror and a pulsatile light producing apparatus. This was the forerunner to today's stroboscope. Stroboscopy uses highspeed flashes of light at a frequency that is slightly lower or higher than the patient's vocal fold vibrations.

The current hospital-based longitudinal investigation looked at 30 cases of benign vocal cord lesions that were clinically diagnosed but did not respond to medical treatment. All of the patients had traditional microlaryngoscopic removal of the benign vocal cord lesion with biopsy under general anesthesia. Videolaryngostroboscopy, acoustic analysis, and
VHI were used to examine these patients after 2 weeks, 6 weeks, and 12 weeks after surgery, and the results were recorded.

The presentstudy covered a total of 30 patients. The participants' ages ranged from 18 to 55-years-old, with a median age of 37.41 years. With $73.3 \%$ male patients and a male to female ratio of $2.75: 1,22$ patients were diagnosed with unilateral lesion $(13,43.3 \%$ right sided, 09, 30.0\%left sided) and $08(26.7 \%)$ patients were diagnosed with bilateral lesion. Hoarseness affects $73.3 \%$ of patients, whereas dysphonia affects $26.7 \%$. Smokers made up $60.0 \%$ of the patients, while non-smokers made up $40.0 \%$. Vocal cord nodules accounted for $56.7 \%$ of the benign lesions found in the study, followed by polyps $(26.7 \%)$, cysts $(10.0 \%)$, and vocal cord hemangioma $(6.6 \%)$.

This finding was in line with $G$ Thomas et al.,'s ${ }^{4}$ age range (18-62 years). In their investigation, Thomas et al., found that 22 patients (15 on the right cord and seven on the left) had unilateral lesions while eight patients had bilateral lesions. $^{4}$

In their study, Mobarsa et al., found that vocal nodules (46.67\%) were the most prevalent benign vocal cord lesion, followed by polyps $(36.67 \%)$, cysts $(13.33 \%)$, and vocal cord hemangioma $(3.33 \%){ }^{5}$

The pre-operative mean fundamental frequency for male patients was 150.23 , with a statistically significant improvement $(\mathrm{P}=0.002)$ to 176.45 during the 12 -week follow-up. Females, on the other hand, had no significant variation in fundamental frequency. The shimmer, which

\section{Table 2:Voice acoustic parameters}

\begin{tabular}{|c|c|c|c|c|c|c|c|c|c|}
\hline \multirow[t]{2}{*}{ Voice Acoustic Parameters } & \multicolumn{2}{|c|}{ Preoperative } & \multicolumn{2}{|c|}{$2^{\text {nd }}$ week } & \multicolumn{2}{|c|}{$6^{\text {th }}$ week } & \multicolumn{2}{|c|}{$12^{\text {th }}$ week } & \multirow[t]{2}{*}{$P$ value } \\
\hline & Mean & $\pm S D$ & Mean & $\pm S D$ & Mean & $\pm S D$ & Mean & $\pm S D$ & \\
\hline Fundamental Frequency $(\mathrm{Hz})$ Male & 150.23 & \pm 18.77 & 154.23 & \pm 21.22 & 163.85 & \pm 18.93 & 176.45 & \pm 21.44 & 0.002 \\
\hline $\begin{array}{l}\text { Fundamental Frequency } \\
(\mathrm{Hz}) \text { Female }\end{array}$ & 203.12 & \pm 15.25 & 209.41 & \pm 18.32 & 217.45 & \pm 23.55 & 231.22 & \pm 20.31 & 0.078 \\
\hline Jitter (\%) Modal/e/ & 2.45 & \pm 0.41 & 1.92 & \pm 0.18 & 1.32 & \pm 0.17 & 0.87 & \pm 0.11 & $<0.0001$ \\
\hline Shimmer (\%) Modal/e/ & 8.95 & \pm 1.07 & 7.35 & \pm 1.02 & 5.44 & \pm 0.33 & 3.12 & \pm 0.97 & $<0.0001$ \\
\hline $\begin{array}{l}\text { Harmonic-to-noise ratio } \\
\text { (dB) Modal/e/ }\end{array}$ & 11.88 & \pm 1.89 & 14.52 & \pm 1.77 & 15.41 & \pm 1.34 & 16.10 & \pm 1.77 & $<0.0001$ \\
\hline
\end{tabular}

Table 3:Outcome according to Voice Handicap Index Score

\begin{tabular}{|c|c|c|c|c|c|c|c|c|}
\hline \multirow[t]{2}{*}{ Voice Handicap Index } & \multicolumn{2}{|c|}{ Pre-operative } & \multicolumn{2}{|c|}{$2^{\text {nd }}$ week } & \multicolumn{2}{|c|}{$6^{\text {th }}$ week } & \multicolumn{2}{|c|}{$12^{\text {th }}$ week } \\
\hline & No & $\%$ & No & $\%$ & No & $\%$ & No & $\%$ \\
\hline Severe & 18 & 60.0 & 5 & 16.7 & 0 & 0.0 & 0 & 0.0 \\
\hline Moderate & 12 & 40.0 & 10 & 33.3 & 9 & 30.0 & 2 & 6.7 \\
\hline Mild & 0 & 0.0 & 15 & 50.0 & 21 & 70.0 & 28 & 93.3 \\
\hline Total & 30 & 100.0 & 30 & 100.0 & 30 & 100.0 & 40 & 100.0 \\
\hline Statistical Inference & \multicolumn{8}{|c|}{$\begin{array}{c}\text { Chi-square: } 71.1994 \\
\mathrm{P}<0.0001\end{array}$} \\
\hline
\end{tabular}


had a mean value of 8.95 , improved to 5.83 , which was statistically significant $(\mathrm{P}=0.0001)$. The jitter improved statistically significantly after surgery, with a pre-operative value of 2.45 and a post-operative value of 0.87 . Preoperatively, the harmonic to noise ratio was 11.88 , with a statistical improvement $(\mathrm{P}=0.0001)$ to 16.10 .

In a study of 905 patients, Preciado et al,, found that the average f0 for 589 females was 228.7 hertz in those without dysphonia and 216.9 hertz in those with dysphonia, while the average f0 for 316 males was 154.7 hertz in those without dysphonia and 139.1 hertz in those with dysphonia. Voice analysis performed after treatment showed significant improvement. ${ }^{6}$

The voice handicap index was assessed both before and after surgery. The outcomes were examined and contrasted. Preoperatively, $40 \%$ (12) of patients had a moderate handicap index, while $60.0 \%$ (18) had a severe handicap index. There was an improvement after the surgery. At the second follow-up, $33.3 \%$ (10) of patients had moderate handicap scores, $50.0 \%$ (15) had mild handicap scores, and $16.7 \%$ (5) had severe handicap scores. At the six-week follow-up, 21 patients $(70.0 \%$ ) had light handicaps and 30.0\% (9) had intermediate handicaps. Finally, during the 12-week follow-up, we discovered that $93.3 \%$ (28) of the patients had mild handicap scores and only $6.7 \%$ (2) had moderate handicap scores, a significant difference $(\mathrm{P}=0.0001)$.

Our findings were similar to those of Thomas, who found significant reductions in total scores as well as functional and physical subscales after surgery. Cheng and $\mathrm{Woo}^{7}$ looked at 21 patients and found that pre- and post-operative VHI values differed significantly.

\section{Limitations of the study}

Our study doesn't cover malignant lesions of the vocal cord and patients were followed only for 12 weeks post operatively.

\section{CONCLUSION}

MLS is an effective procedure to provide improvement in speech among patients with benign vocal cord lesion. The use of a combination of stroboscopic analysis, acoustic analysis, and the VHI score in the pre- and post-operative evaluation of benign vocal cord lesions is beneficial. In terms of diagnosis, management, and follow-up, all three techniques are complementary.

\section{ACKNOWLEDGMENT}

We acknowledge our department seniors and colleagues for guiding in this study and also the patients who participated in this study

\section{REFERENCES}

1. Gray SD, Hammond E and Hanson DF. Benign pathologic responses of the larynx. Ann OtolRhinolLaryngol. 1995;104(1):13-18.

https://doi.org/10.1177/000348949510400103

2. Scalco AN, Shipman WF and Tabb HG. Microscopic suspension laryngoscopy. Ann OtolRhinolLaryngol. 1960;69:1134-1138.

https://doi.org/10.1177/000348946006900421

3. Courey MS, Gardner GM, Stone RE and Ossoff RH. Endoscopic vocal fold microflap: A three-year experience. Ann OtolRhinolLaryngol. 1995;104(1):267-273.

https://doi.org/10.1177/000348949510400402

4. Thomas G, Mathews SS, Chrysolyte SB and Rupa V. Outcome analysis of benign vocal cord lesions by videostroboscopy, acoustic analysis and voice handicap index. Indian J Otolaryngol Head Neck Surg. 2007;59(4):336-340. https://doi.org/10.1007/s12070-007-0096-0

5. Mobarsa V, Samdani SK and Gurjar VS. Outcome analysis of microlaryngeal surgery for benign lesions of vocal cord using videostroboscopy and voice handicap index. Indian J Otolaryngol Head Neck Surg. 2019;71Suppl 1:S327-S332.

https://doi.org/10.1007/s12070-018-1300-0

6. Preciado J, Pérez $\mathrm{C}$, Calzada $\mathrm{M}$ and Preciado $\mathrm{P}$. Function vocal examination and acoustic analysis of 905 teaching staff of La Rioja, Spain. ActaOtorrinolaringol Esp. 2005;56(6):261-272.

https://doi.org/10.1016/S0001-6519(05)78612-X

7. Cheng $\mathrm{J}$ and Woo P. Correlation between the voice handicap index and voice laboratory measurements after phonosurgery. Ear Nose Throat J. 2010;89(4):183-188. https://doi.org/10.1177/014556131008900411

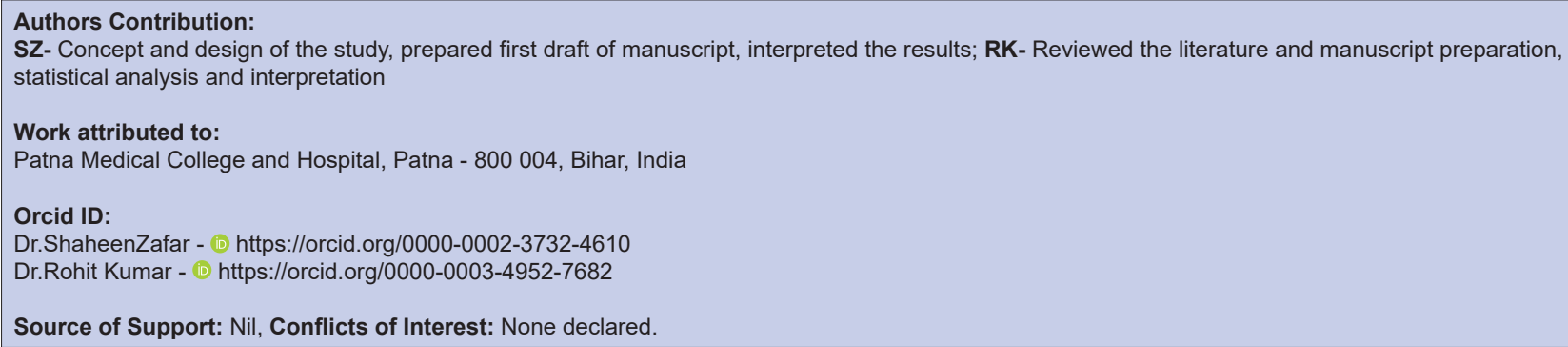

